\title{
Investigating Road Safety Management Systems in the European Countries: Patterns and Particularities
}

\author{
Victoria Gitelman ${ }^{1}$, Etti Doveh ${ }^{2}$ \\ ${ }^{1}$ Transportation Research Institute, Technion-Israel Institute of Technology, Haifa, Israel \\ ${ }^{2}$ Technion Statistical Laboratory, Haifa, Israel \\ Email: ^trivica@technion.ac.il, ierde01@ie.technion.ac.il
}

How to cite this paper: Gitelman, V. and Doveh, E. (2016) Investigating Road Safety Management Systems in the European Countries: Patterns and Particularities. Journal of Transportation Technologies, 6, 378-404.

http://dx.doi.org/10.4236/jtts.2016.65032

Received: August 4, 2016

Accepted: September 27, 2016

Published: September 30, 2016

Copyright $\odot 2016$ by authors and Scientific Research Publishing Inc. This work is licensed under the Creative Commons Attribution International License (CC BY 4.0).

http://creativecommons.org/licenses/by/4.0/

\begin{abstract}
A road safety management (RSM) system can be defined as "a complex institutional structure that involves cooperating and interacting bodies which support the tasks and processes necessary for the prevention and reduction of road traffic injuries". RSM should promote the road safety progress of the country. However, the details of this relationship are generally lacking. This study explored the RSM systems in European countries based on the information collected through interviews with experts and officials, in each country, and using a "good practice" criteria questionnaire. The dataset included 14 countries with fifty items related to five RSM areas: institutional organization; policy formulation and adoption; policy implementation and funding; monitoring and evaluation; scientific support, information and capacity building. Cluster analyses and correlations were used to identify country groups with similar RSM components, to recognize typical RSM structures if available and to examine the relationship between RSM and road safety performance of the countries. The findings showed that all the countries are different when RSM systems are considered as a whole, making it impossible to identify typical RSM structures or a single best working model at a national level. However, it is possible to compare countries when the RSM areas are considered separately, where the clusters of countries recognized by the study present the patterns common for those European countries. Across the analyses, a number of countries with a consistently higher and lower availability of the RSM components were identified, enabling a final countries' ranking into a number of groups. The latter actually reflects the level of RSM in the country, in terms of its correspondence to the "good practice" criteria. A further analysis indicated a positive correlation between the higher level of the RSM system and better safety performance of the countries.
\end{abstract}




\section{Keywords}

Road Safety Management, Good Practice, Country, Clustering, Questionnaire

\section{Introduction}

Road safety management (RSM) implies systematic work to ensure continuous improvement in road safety [1] [2]. The need for effective RSM is widely recognized today due to the global burden of road trauma for society and public health; preventability of major parts of road fatalities and severe injuries, and the availability of knowledge on measures and interventions that can be applied to achieve the results [3] [4].

An RSM system can be defined as "a complex institutional structure that involves cooperating and interacting bodies which support the tasks and processes necessary for the prevention and reduction of road traffic injuries" [5]. By definition, an RSM system should meet a number of "good practice" criteria spanning the entire policy-making cycle, from agenda setting to policy formulation, adoption, implementation and evaluation, and include an efficient structure and smooth processes, in order to enable evidence-based policy-making.

A number of studies aspired to describe the main components of effective RSM. The development and implementation of a road safety program is recognized as a major RSM component [1] [3]. The OECD report [6] identified the main steps of a planning procedure for developing and implementing road safety programs, including: formulation of a vision, problem analysis and target setting, developing countermeasures and conducting socio-economic evaluations for selecting the best alternative, establishing and implementing the program, and further systematic monitoring of the program's performance. There are strong indications that the existence of sound road safety programs together with quantified targets contribute positively to road safety performance of the countries [7]-[9].

ETSC [7] reviewed road safety strategies and best practice activities in a number of European countries with the purpose to set out a methodological approach to the effective development and implementation of national road safety policies. The study suggested a checklist of 22 prerequisites for successful road safety work but concluded that no stringent recipes were available for road safety performance success and that the strategy should be adapted to a country's conditions.

The OECD report [1] underlined that the "results focus" is a pivotal characteristic of an effective RSM system. A country's "result focus" is a pragmatic specification of its "ambition" to improve road safety and the means agreed to achieve this ambition [4]. According to OECD [1], the results focus requires clear identification of: a lead agency; the core group of government ministries and agencies to be involved, their roles and responsibilities; and the performance targets in terms of institutional outputs and intermediate and final outcomes to be achieved within a defined strategy. In this context, a Safe-System approach is recommended today for adoption by the developed countries 
[1]. It is focused on system-wide interventions, long-term elimination of deaths and serious injuries and shared responsibility, where the "blame the victim" culture is superseded by "blaming the traffic system" which throws the spotlight on authorities' accountability.

More recent overview reports on RSM [1] [4] emphasized the point previously made by some researchers [10] [11] that the limits of improved road safety performance were shaped by the capacity of the RSM system operating in a country. The system's institutional capacity may constrain the implementation of efficient interventions, while the subsequent results may fall short of what is technically feasible with any particular set of road safety interventions.

The evolution of the RSM system which should overcome such constraints was recommended by Bliss \& Breen [4]. This approach highlights the importance of addressing road safety management weaknesses and the need for effective institutional management as a pre-requisite for successful results-focused interventions. According to this approach, safety is compared with other goods and services and the production process is viewed as a management system with three levels: institutional management functions which produce interventions, which in turn produce results. The institutional management functions include [4]: results focus (a strategic orientation that links all actual and potential interventions with results); coordination; legislation; funding and resource allocation; promotion (communication of road safety); monitoring and evaluation; research and development and knowledge transfer. It can be noted that the institutional management functions (as defined by [4] and cited by [1]) have much in common with the prerequisites for successful road safety work mentioned by ETSC [7]. In both cases the emphasis is on the components enabling systematic work and progress through the entire policy-making cycle.

At the same time, the "good practice" may have different forms in different countries, depending on local conditions. Case-studies indicated that effective management can be achieved with varied lead agency structural and procedural forms [4]. Actual developments showed that countries have evolved their own RSM systems over time and, although the systems are different, it was observed that many European countries did reach the 2010 European road safety target [12].

In a broad sense, "good practice" can be defined as "building up the RSM system and developing the activities it supports (processes and tasks) so as to ensure that the expected road safety outputs are effectively obtained and are as efficient in reducing road crashes and injuries as we can make them, given the present state of road safety knowledge" [5]. Thus, based on the available knowledge on RSM [1] [4] [10] and assuming a diversity of components in the RSM processes, an RSM investigation model was proposed within the DaCoTa research project [5].

The investigation model was designed for studying the various aspects of actual road safety policy-making and management processes in Europe [5]. It included a description of the policy-making tasks (agenda setting, policy formulation, adoption, implementation and evaluation) and accompanying management processes (inter-sectoral 
coordination, consultation of stakeholders, knowledge production and use, capacity building), which were considered in the context of road safety management structures and outputs and related to pre-conditions-Figure 1. Then, "good practice" elements were defined for each component, enabling a "diagnosis" of a country in accordance with the presence or absence of certain conditions/ elements in the RSM system. On the basis of the investigation model, an extensive questionnaire was developed [5] and applied for collecting information from 14 countries, by means of personal interviews with independent experts and governmental representatives, in each country.

The qualitative analysis of the questionnaire responses enabled to draw a reliable "profile" for each country and to perform in-depth country comparisons by selected key items [13]; more details on the topic can be found in Papadimitriou et al. [14]. This paper presents the results of quantitative analyses which were carried out on the questionnaire responses, using statistical methods and aiming to identify patterns and rankings of countries, regarding both the RSM characteristics, and the relationship between RSM and road safety performance. Such quantitative and comparative analyses were not carried out in previous research. The novelty of this study lies in the possibility of providing new insights with regards the common components of RSM in the European countries as well as their differences, based on the detailed characteristics of the RSM systems. The data analyses applied in this study actually examined the hypothesis raised (but not statistically proven) by previous research that "good practice" RSM may have different forms in different countries [4] [5]. In addition, this study examined empirical evidence concerning a relation between "good practice" RSM and a country's safety performance which was not provided by previous research [1] [4] [7].

\section{Methodology}

This study aimed to investigate the RSM systems in the developed countries, based on the RSM questionnaire responses. Statistical methods were applied to the answers provided to the RSM questionnaire in order to identify country groups with similar availability of certain RSM components, to recognize typical RSM structures, if available, in

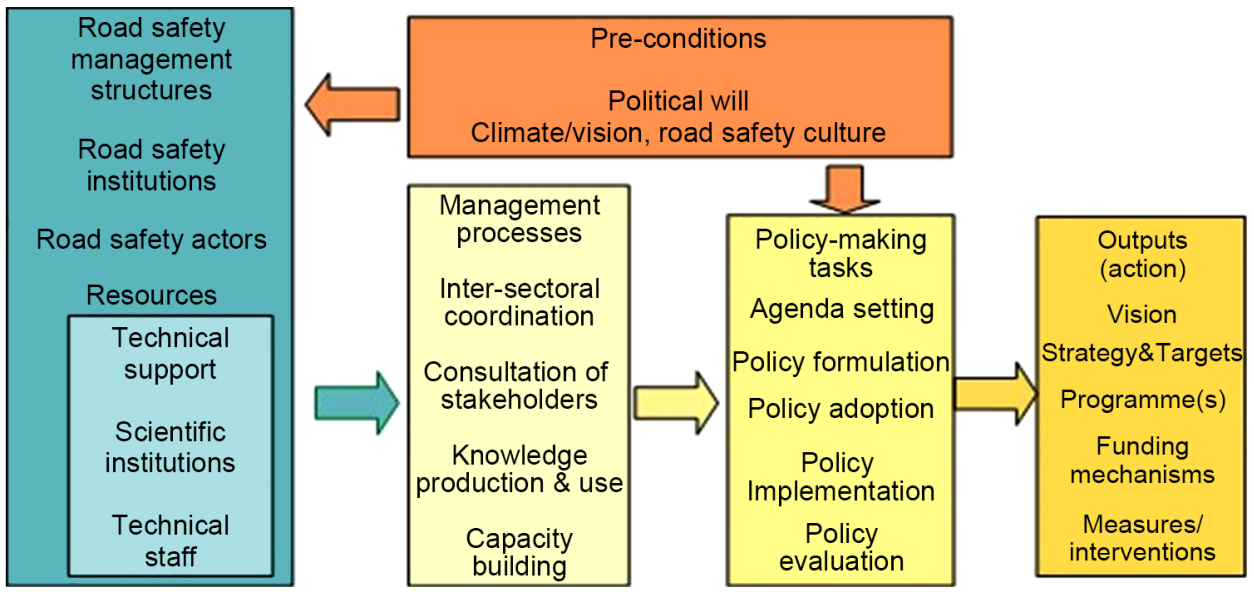

Figure 1. Road safety management components. Source: [5]. 
Europe and to examine the relationship between the RSM characteristics and countries' safety performance.

\subsection{Data Preparation}

Two groups of road safety professionals were targeted by the RSM questionnaire: 1) independent experts-road safety researchers or scientists who may contribute to policy but do not have a decision-making role and could offer a view of the RSM systems in place, and 2) government representatives-road safety practitioners who were directly involved in policy and decision-making. The questionnaire was filled in for 14 countries, including: Austria, Belgium, Finland, France, Greece, Ireland, Israel, Italy, Latvia, the Netherlands, Poland, Spain, Switzerland and the United Kingdom. In total, 14 independent expert responses and 12 governmental responses were collected [14]. A general assumption of the study was that governmental and expert visions of the RSM situation in the country would not necessarily be identical (that is why both sides were asked to fill in the questionnaire). Therefore, separate analyses of expert versus governmental responses were conducted.

The questionnaire applied for investigating RSM systems of the countries related to five main areas of RSM [5]:

- Institutional organisation, coordination and stakeholders' involvement;

- Policy formulation and adoption;

- Policy implementation and funding;

- Monitoring and evaluation;

- Scientific support and information, capacity building.

The RSM questionnaire was structured in five parts, respectively, and contained a total of 50 questions (variables) as detailed in Table A1, in Appendix. The data preparations included: coding the response values, data imputations and preliminary data analysis to exclude the variables with a low potential for contribution to the analysis. All responses were coded using the following scale: 1 for "yes", 2 for "no", 1.5 for "unknown" (in the latter case, it was assumed that lack of information not necessarily indicates the absence of a certain RSM component). In addition, some questions were subdivided into several sub-questions in the original questionnaire, where the final answer on such a question should account for all the sub-answers. For such composite questions (see Table A1), a single score was estimated, which corresponds to the mean value of answers provided for all the sub-questions. For some questions, namely 7, 12, 13, answers were missing. These were imputed using the MI procedure of SAS 9.2. As a result, for each response, a list of 50 coded values was produced; the final dataset based on the expert responses is presented in Table A1.

Further examination of the dataset revealed that the answers to some questions hardly varied between the countries, i.e. there were 12 to 14 identical answers for the 14 countries. Given the high consensus among the countries concerning the availability of the RSM components addressed by these questions, they were termed "consensus variables". They would not really contribute to the countries' grouping, and thus were ex- 
cluded from the analysis ( 7 questions in total, see Table A1); for the majority of these questions "yes" was a common answer among the countries, except for question 23 for which the typical answer was "no".

Due to the small samples of the responses (related to the questionnaire size) a separate analysis was carried out for each part of the questionnaire.

\subsection{Analysis Methods}

Clustering methods were applied in the study for identifying country groups with similar RSM components. Cluster analysis is an exploratory data analysis tool for organizing observed items into meaningful groups, or clusters, based on combinations of independent variables, which maximizes the similarity of cases within each cluster while maximizing the dissimilarity between the groups [15] [16].

A wide number of clustering algorithms exists in the literature (e.g. [17]). One should be aware that different clustering algorithms may give us different results on the same data. Hence, in the current study we decided to use several approaches, in order to get an impression about the stability of our solution, over the methods applied. Another known problem in clustering analysis is how to find the right number of clusters. Typically, no "best" solutions for the problem of determining the number of clusters to extract can be suggested. In most cases, a reasonable approach is to select the number of clusters that gives the most natural partition according to some distance measure, or visual plot. Thus, in the current study, several methods for selecting the clusters' number were tried, to demonstrate the solution. If the results of several trials are consistent, this strengthens our belief in the validity of the obtained country groups.

To cluster the countries into similar groups we applied two types of clustering methods commonly used in cluster analysis: hierarchical clustering and $k$-means clustering. In a hierarchical classification, the data are not partitioned into a particular number of groups at a single step. Instead, the classification consists of a series of partitions that may run from a single "cluster" containing all items to $n$ clusters, each containing a single item. Hence, this method produces partitions by a series of successive fusions of the $n$ items into groups [18].

The criterion used for choosing the pair of clusters to merge at each step was the Ward minimum variance criterion, which minimizes the total within-cluster variance [19]. To be specific, the objective at each step of the Ward method, is to minimize the increase caused by the fusion of two clusters in the value of $E$, that is given by

$E=\sum_{m=1}^{g} E_{m}$, where $E_{m}=\sum_{l=1}^{n_{m}} \sum_{k=1}^{p_{k}}\left(x_{m l, k}-\bar{X}_{m, k}\right)^{2}$, in which $\bar{X}_{m, k}=\frac{1}{n_{m}} \sum_{l=1}^{n_{m}} X_{m l, k}$.

The latter is the mean of $m$ cluster for the $k$ variable, $x_{m l l k}$ is the score on the $k$ variable $(k=1, \cdots, p)$ for the $l$ item $\left(l=1, \cdots, n_{m}\right)$ in the $m$ cluster $(m=1, \cdots, g)$.

The $k$-means clustering method seeks to partition $n$ items in a set of multivariate data into $k$ groups, i.e. $G_{1}, G_{2}, \cdots, G_{k}$ where $G_{i}$ denotes the set of $n_{i}$ items in the $i$ group and $k$ is given, by minimizing a numerical criterion, lower values of which indicate a "better" solution. The most commonly used criterion is the within-group sum of squares 
(WGSS) over all variables; explicitly, we used the following criterion:

$$
\text { WGSS }=\sum_{j=1}^{q} \sum_{l=1}^{k} \sum_{i \in G_{I}}\left(x_{i j}-\bar{x}_{j}^{(l)}\right)^{2} \text {, where } \bar{x}_{j}^{(l)}=\frac{1}{n_{i}} \sum_{i \in G_{I}} x_{i j} .
$$

The latter is the mean of the items in group $G_{l}$ on variable $j$ [18]. This function aims to subdivide the items into $k$ groups so that the sum of squares of distances from the items to the assigned cluster centers is minimized.

To apply the methods, we used both the cluster library of R software, and CLUSTER procedure of SAS 9.2. Applying hierarchical cluster analysis with the Ward method, for selecting the number of clusters required, two values were used: 1$)$ the pseudo- $F$ statistic, and 2) the pseudo $T$-squared statistic, where both values are plotted against the number of clusters. The pseudo- $F$ statistic is intended to capture the "tightness" of clusters, where it is estimated as a ratio of the mean sum of squares between groups to the mean sum of squares within group. Larger numbers of the pseudo- $F$ usually indicate a better clustering solution. Using the pseudo $T$-squared statistic, one should look at the plot from right to left until finding the value markedly larger than the previous value, and then move to the right in the plot by one step in the cluster history. To consider the clusters' composition, a dendrogram (a tree diagram created by the method) was applied. The clustering height appearing on the dendrogram is the value of the criterion associated with the Ward error sum of squares, for the particular classification.

Applying the $k$-means method, a plot of the WGSS values by the number of clusters helps to determine the appropriate number of clusters. Using the plot, the analyst should look for a kink in the sum-of-squares curve to locate the optimal number of clusters [16].

A more robust version of the $k$-means is called PAM (Partitioning Around Medoids), where clustering of the data into $k$ clusters "around medoids" is done. A Silhouette plot of the items classified is produced, providing a succinct graphical representation of how well each item lies within its cluster. The Silhouette method was introduced by Rousseeuw [20], and it is considered as a method of interpretation and validation of clusters of data. Looking at the Silhouette plot of items the following clues can be applied: a Silhouette close to one means that the datum is appropriately clustered; a Silhouette close to negative means that it would be more appropriate to cluster the item in its neighboring cluster; a Silhouette near zero means that the datum is on the border of two natural clusters. The average of Silhouettes of a cluster is a measure of how tightly all the data in the cluster are grouped. Hence, the average Silhouette of the entire dataset is a measure of how appropriately the items have been clustered.

To summarise, considering each part of the RSM questionnaire for the countries' clustering, two methods were applied: the Ward and the $k$-means. To determine the number of clusters required we considered: the pseudo $F$ - and the pseudo $T$-squared statistics of the Ward's method, and the plot of the $k$-means. To examine the composition of the clusters extracted we compared: 1) the classification tree (dendrogram) produced by the Ward method, 2) the groups created by the $k$-means, and 3) the clusters' Silhouette produced by the PAM-method. If the composition of the clusters created by 
different methods is close, one can state that a stable countries' classification into homogeneous groups has been achieved. All the variable values were standardized before clustering, by subtracting the mean of each variable and dividing by its standard deviation.

The country groupings were produced using expert responses. Then, they were compared with the results of similar classifications based on the governmental responses. Furthermore, a final country ranking according to availability of the RSM components was considered and a statistical link between the country ranking and their safety performance was examined (using fatality rates per population). The latter applied a Spearman correlation coefficient considering the fatality rate as a dependent variable, where country clusters served as an independent one; the CORR procedure of SAS was applied.

\section{Results and Discussion}

\subsection{Grouping Countries Based on the Analysis of Expert Responses}

\subsubsection{Institutional Organisation, Coordination and Stakeholders' Involvement}

Part 1 of the RSM questionnaire included 9 questions (see Table A1). An initial analysis revealed a high correlation (Pearson correlation coefficients of $0.55-0.81, \mathrm{p}<0.05$ ) between the pairs of variables (question responses) such as: 7 and 9, 2 and 6, 1 and 5 . These pairs of variables were united and averaged, reducing the dimensionality of the analysis. Hence, for the countries' clustering 6 variables were applied.

Using all the metrics introduced in Sec. 2.2 (the plots of the pseudo $F$ - and the pseudo $T$-squared statistics of the Ward method and the plot of the k-means' within groups sum of squares), four clusters were found to be an appropriate choice for the data considered. The classification results received by different methods are presented on Figures 2(a)-(c). Figure 2(d) characterizes the clusters in terms of the mean values of original variables (the RSM components' availability) by cluster.

The classification results in Figure 2 show that both the Ward and the Silhouette methods identified two homogeneous groups of countries: NL, UK, AT, CH and IE, PL, IT, LV (see country abbreviations in Table A1). Additional stable country groups were FI, FR and EL, ES, which were recognized by all the methods. In contrast, IL and BE seem to be different from other countries where they changed the group depending on the method of clustering. However, according to the Ward method, IL was found to be similar to the FI, FR subgroup, where according to the $k$-means BE was attached to that subgroup. Thus, the four clusters' composition based on the Silhouette method, where IL and BE were attached to the FI, FR country subgroup seemed to provide a reasonable summary of the results produced by different methods.

The countries' groups recognized using the Part 1 components of the RSM questionnaire were as follows:

1 (FI, FR, IL, BE) - includes countries definitely having a high level inter-sectoral decision-making institution to prepare policy orientations and a technical inter-sectoral road safety institution to coordinate policy formulation and implementation. In addition, 


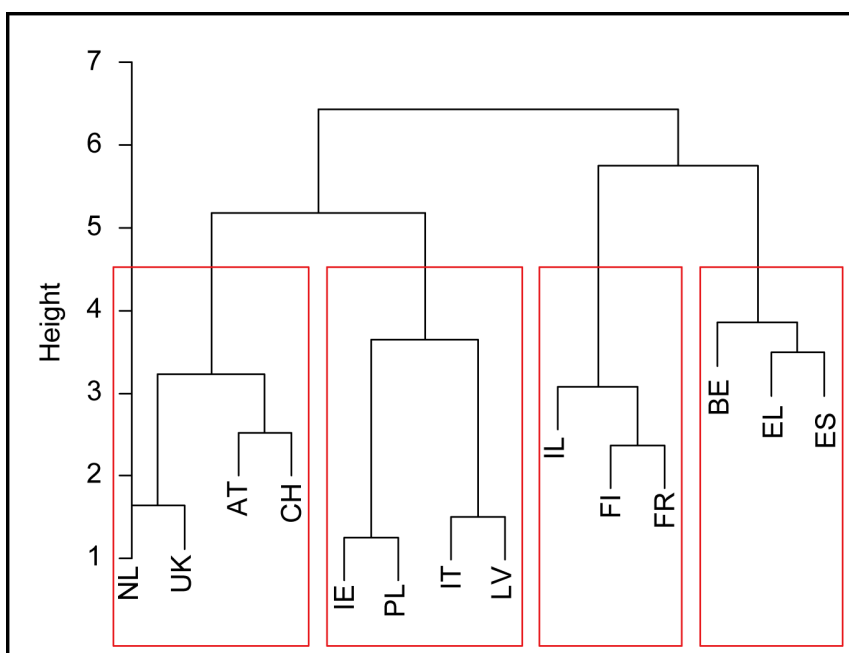

(a)

1 IL IE PL

$2 \mathrm{BE} \mathrm{FI} \mathrm{FR}$

3 IT LV CH NL UK

4 AT EL ES
Silhouette plot of pam $(x=x d a t, k=i)$

$\mathrm{n}=14$

4 clusters $C_{i}$ $\mathrm{j}: \mathrm{n}_{\mathrm{j}}$ ave $_{\mathrm{i} \in \mathrm{C}} \mathrm{S}_{\mathrm{i}}$

NL

UK

AT

$\mathrm{CH}$

FR

$\mathrm{FI}$

BE

IL

EL

ES

IE

LV

PL

IT

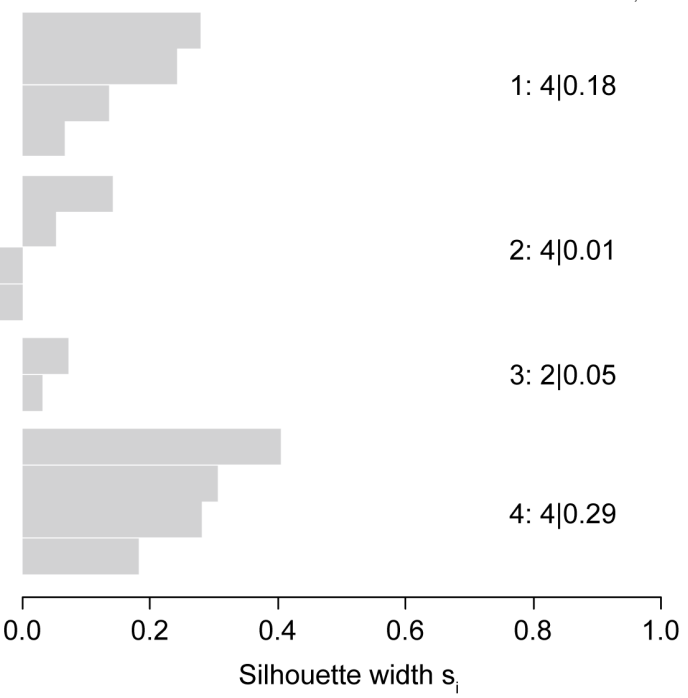

(c) (b)

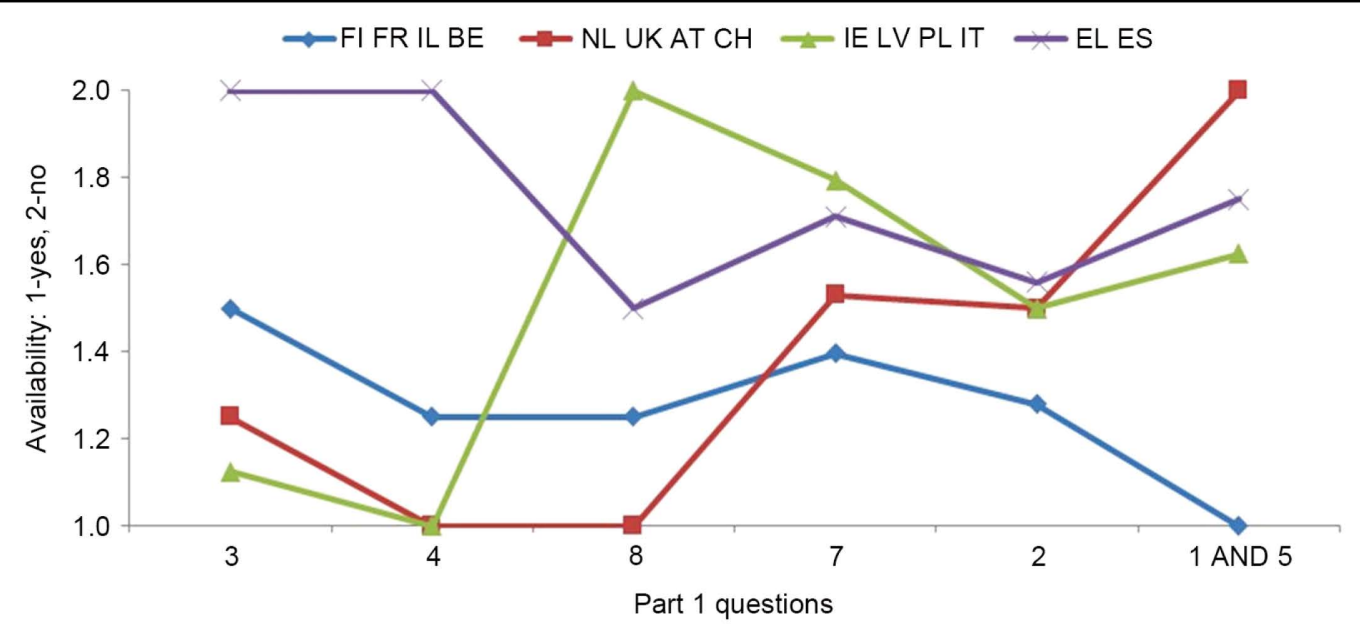

(d)

Figure 2. Result of countries' clustering using the "Institutional organization" characteristics: (a) a cluster dendrogram produced by the Ward method, (b) groups created by the k-means, (c) clusters' Silhouette produced by the PAM-method, (d) mean values of availability of the RSM components, by clusters of countries.

most of other "Institutional organization" RSM components tend to be available in these countries;

2 (NL, UK, AT, CH) - includes countries definitely having a lead agency formally appointed to take responsibility for road safety and in which an institutional structure for the consultation of stakeholders was formally established. Also, in these countries, Parliament is usually involved in adopting road safety orientations. At the same time, a high level inter-sectoral decision-making institution to prepare policy orientations and 
a technical inter-sectoral road safety institution to coordinate policy formulation and implementation were not established in these countries;

3 (IE, LV, PL, IT) — contains countries having a lead agency, where Parliament is typically involved in adopting road safety orientations. At the same time, the countries do not have the institutional structure for the consultation of stakeholders, where the vertical coordination tends to be absent and the legislative instruments defining inter-sectoral road safety management functions are not periodically reviewed;

4 (EL, ES) - comprises countries for which the majority of the "Institutional organization" RSM components tend to be lacking. In particular, the countries do not have a lead agency appointed to take responsibility for road safety, and Parliament is not involved in adopting road safety orientations.

\subsubsection{Policy Formulation and Adoption}

Part 2 of the RSM questionnaire included 8 variables (excluding the "consensus" variables, see Table A1). An initial analysis found certain correlations between the pairs of variables-responses to questions: 19 and 20, 12 and 13 (Pearson coefficients of 0.61 $0.73, \mathrm{p}<0.05)$, as well as 16 and $18(0.45, \mathrm{p}=0.11)$. The values of these pairs were averaged and for the countries' clustering 5 variables were left.

Using the pre-defined metrics, four clusters seemed to be an appropriate choice for the Part 2 data. The classification results received by different methods are presented in Figure 3, together with the mean values of availability of RSM components, by country clusters. Considering the classification results in Figure 3, the following observations are evident: the Ward and the $\mathrm{k}$-means methods produced identical results as to the composition of the four country groups; the Silhouette method also identified two homogeneous groups of countries, which are similar to the results of the two other methods. These groups are: FR, EL, IT, BE and IE, PL, UK, LV. However, the two other groups of countries recognized by other methods were not fully supported by this method, especially concerning the position of AT, CH, ES.

Accounting for the identical results produced by two methods (out of three examined), the countries' groups recognized using the Part 2 components of the RSM questionnaire were as follows:

1 (AT, CH) -includes the countries in which both local and regional authorities are consulted as to the part they are called to play in the national road safety policy where their local/regional programs are integrated into the national road safety policy;

2 (ES, FI, IL, NL)-comprises the countries in which a national medium-term road safety program was elaborated and adopted at a high level, and a national "vision" for improved road safety performance in the long-term was set, mostly based on a SafeSystem approach [1]. In addition, in these countries local authorities are usually consulted as to the part they are called to play in national road safety policy but their local programs are not integrated into the national road safety policy;

3 (IE, LV, PL, UK) - contains the countries in which a national medium-term road safety program was elaborated and adopted at a high level, but a national "vision" for improved road safety performance was not set. Also, in these countries, local and 


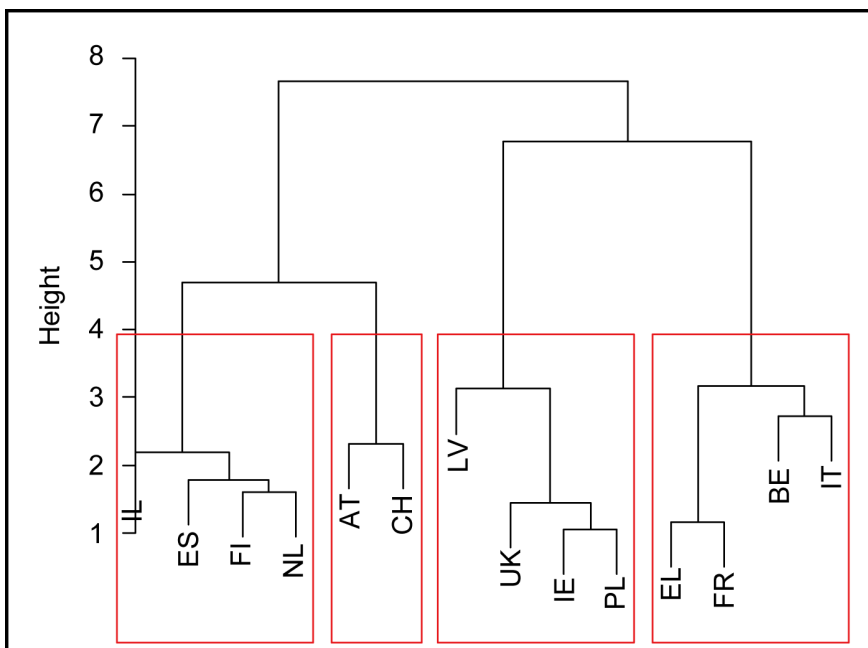

(a)

$1 \mathrm{AT} \mathrm{CH}$

2 BE EL FR IT

3 ES FI IL NL

4 IE LV PL UK
Silhouette plot of pam $(x=x d a t, k=i)$

$\mathrm{n}=14$

4 clusters $\mathrm{C}$

$\mathrm{j}: \mathrm{n}_{\mathrm{j}} \mid \mathrm{ave}_{\mathrm{i} \in \mathrm{C}} \mathrm{S}$

1: $4 \mid 0.14$

2: $4 \mid 0.26$

3: $1 \mid 0.00$

(b)

4: $5 \mid 0.28$

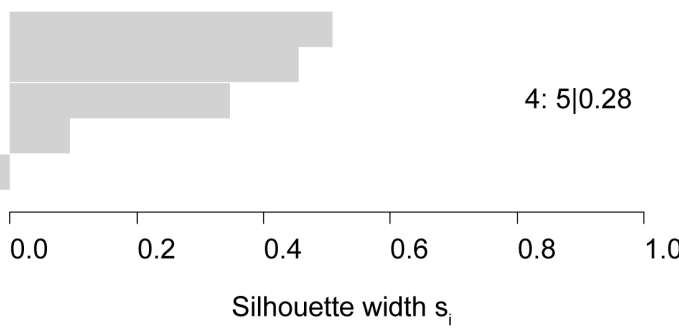

(c)

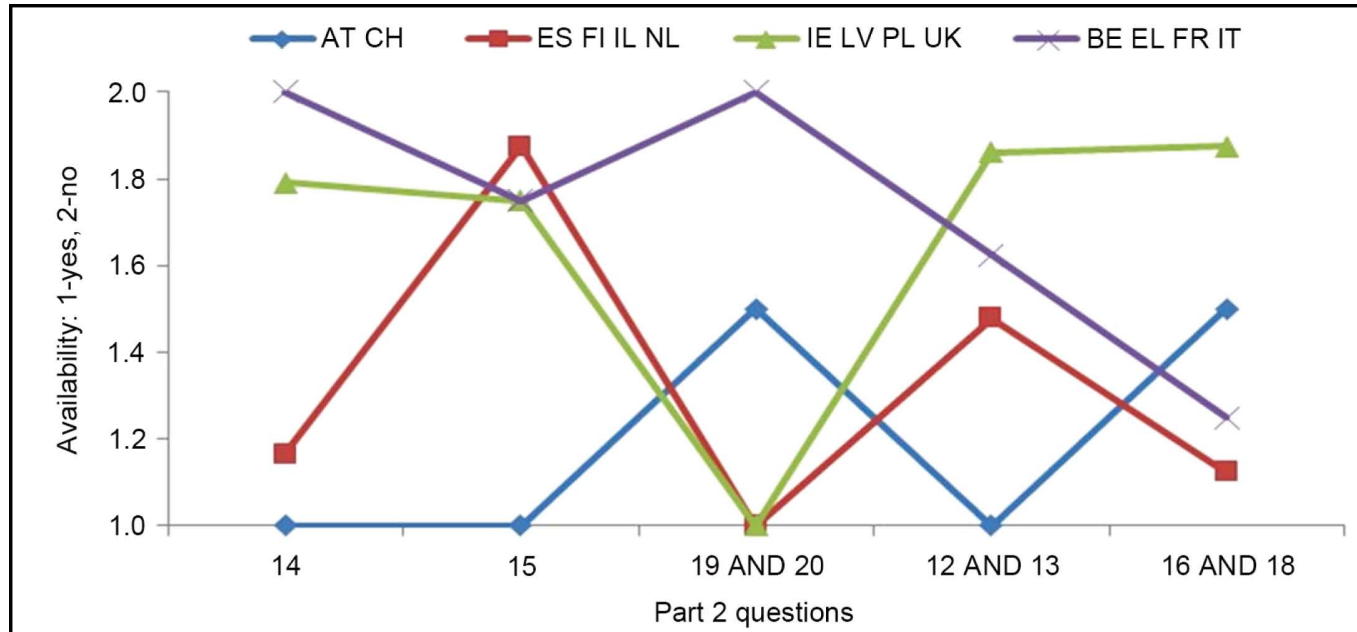

(d)

Figure 3. Result of countries' clustering using the "Policy formulation and adoption" characteristics: (a) a cluster dendrogram produced by the Ward method, (b) groups created by the k-means, (c) clusters' Silhouette produced by the PAM-method, (d) mean values of availability of the RSM components, by clusters of countries.

regional authorities are usually not involved in the road safety policy formulation and adoption;

4 (BE, EL, FR, IT) -includes the countries not having a national medium-term road safety program, where they all have a national long-term "vision" for improved road safety performance. Local authorities are usually not involved in the preparation of the national road safety policy. Similarly, regional authorities are usually not involved in the preparation of the national road safety policy, except for one country (BE). 


\subsubsection{Policy Implementation and Funding}

Part 3 of the RSM questionnaire included 12 variables (excluding a consensus variable, see Table A1). In the preliminary analysis, certain correlations were observed between groups of variables such as responses to questions: 29 - 31 and 24, 26, 32 (Pearson coefficients of $0.63-0.79$ and $0.56-0.90, \mathrm{p}<0.05)$. Both groups were averaged, respectively; for the countries' clustering 8 variables remained.

Considering the plots of the Ward and the k-means' methods for criteria of the number of clusters required, 2, 3 or 6 clusters were possible as an appropriate solution. A further analysis demonstrated higher consistency in the classification results received by different methods for the 6-cluster solution that was selected for further consideration. The classification results received by different methods are presented in Figure 4 as well as the mean values of availability of RSM components, by country clusters.

One can observe that the Ward and the k-means methods produced identical results as to the composition of the six country groups. The Silhouette method also identified four homogeneous groups of countries, which are identical to the results of the two other methods. In addition, countries FR, IT, UK were also recognized by this method as a homogeneous subgroup, yet, inside a larger group of countries. The main difference in the results of this method concerns the position of two countries: AT and BE (which by other methods were seen as a common group).

Summing up the classification results and accounting for the country groups identically recognized by two methods, the countries' groups identified using the "Policy implementation and funding" components of the RSM questionnaire were defined as follows:

$1(\mathrm{CH}, \mathrm{FI})$ - includes countries in which the budget needed for program implementation was estimated; there is a sustainable funding structure for road safety, independent from the Treasury; and training plans were designed to support implementation of the national road safety program. However, the funds allocated on implementation of the program and the human resources needed for it are considered, in these countries, as insufficient. Similarly, a high level decision to ensure availability of a budget for road safety was not taken and there is no budget specifically allocated to road safety from the Treasury;

$2(\mathrm{NL})$ - contains a country in which there is a sustainable funding structure for road safety, independent from the Treasury; training plans were designed to support implementation of the national road safety program; the funds allocated on implementation of the program and the human resources needed for it are considered as sufficient; there is a budget specifically allocated to road safety from the Treasury and related legislative instruments and procedures are regularly reviewed. On the other hand, it was stated that the budget needed for program implementation was not estimated, the government does not allocate the product of fines to road safety activities and there are no formal resource allocation procedures to support RSM tasks and interventions (yet, sufficient budget is available for road safety activities);

3 (ES, IL, LV)-comprises countries in which the budget needed for program implementation was estimated and formal resource allocation procedures to support RSM 

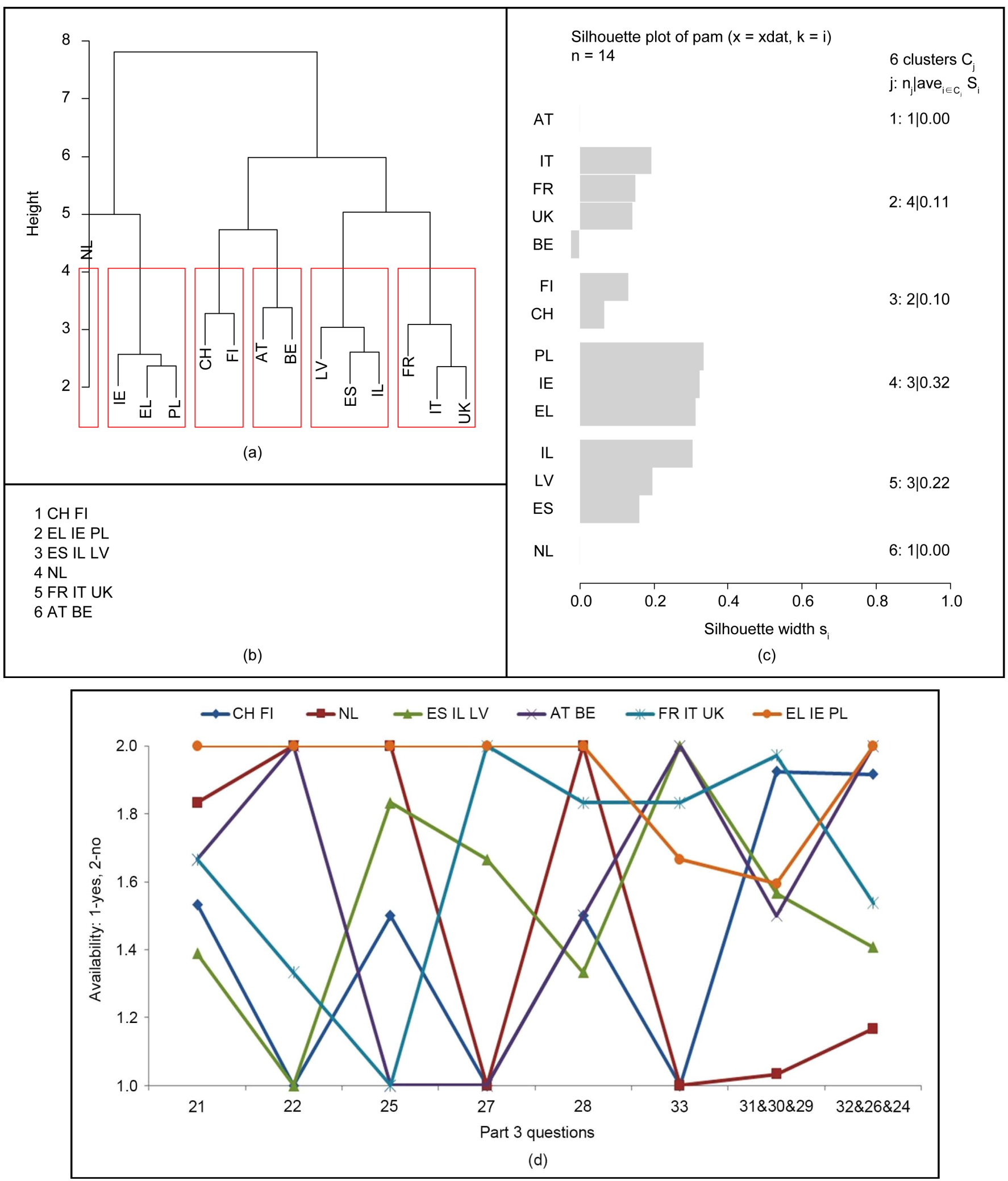

Figure 4. Result of countries' clustering using the "Policy implementation and funding" characteristics: (a) a cluster dendrogram produced by the Ward method, (b) groups created by the k-means, (c) clusters' Silhouette produced by the PAM-method, (d) mean values of availability of the RSM components, by clusters of countries. 
tasks and interventions are mostly available. In addition, for these countries, a budget specifically allocated to road safety from the Treasury tends to be available and relevant legislative instruments and procedures tend to be regularly reviewed. On the other hand, training plans were not designed to support implementation of the national road safety program and the government does not allocate the product of fines to road safety activities;

4 (AT, BE) - includes countries in which the government does allocate the product of fines to road safety activities and there is a sustainable funding structure for road safety, independent from the Treasury. However, the budget needed for program implementation was not estimated, training plans supporting the program were not designed, there is no budget specifically allocated to road safety from the Treasury, and the legislative instruments and procedures are not regularly reviewed;

5 (FR, IT, UK) - comprises countries in which the government allocates the product of fines to road safety activities and the budget needed for the program implementation was mostly estimated. However, as stated by the experts, these countries do not have a sustainable funding structure for road safety, independent from the Treasury; the funds allocated on implementation of the program and the human resources needed for it are considered as insufficient, where concerning the availability of other components the answers tend to be negative;

6 (EL, IE, PL) - includes countries in which experts reported on unavailability of the majority of "Policy implementation and funding" RSM components.

\subsubsection{Monitoring and Evaluation}

Part 4 of the RSM questionnaire included 8 variables (having excluded a consensus variable, see Table A1). In an initial analysis, certain correlations were observed between the pairs of variables such as responses to questions: 36 - 37 and $41-42$ (Pearson coefficients of $0.59-0.75, \mathrm{p}<0.05)$. The values of these pairs were averaged and, consequently, for the countries' clustering 6 variables remained.

The plots of criteria for selecting the number of clusters did not indicate a consistent choice in this case. Based on the $k$-means plot, 2 - 4 cluster solutions were examined. A further analysis demonstrated higher consistency in the classification results received by different methods for the 2-clusters' solution, that was selected for application. Figure 5 presents the classification results received by different methods and the mean value of clusters across the RSM components, based on Part 4 of the questionnaire. One can observe that the Ward and the $k$-means methods produced identical results as to the composition of the two country groups. Similarly, the Silhouette method recognized a homogeneous group of three countries (EL, IT, AT), where PL was classified in another group (yet, the Silhouette plot indicates that PL differs from other countries in the second group).

The countries' groups recognized using the "Monitoring and evaluation" components of the RSM questionnaire were:

1 (BE, CH, ES, FI, FR, IE, IL, LV, NL, UK) - comprises countries in which, mostly, "benchmarking" relative to other (European) countries is used to monitor progress of 


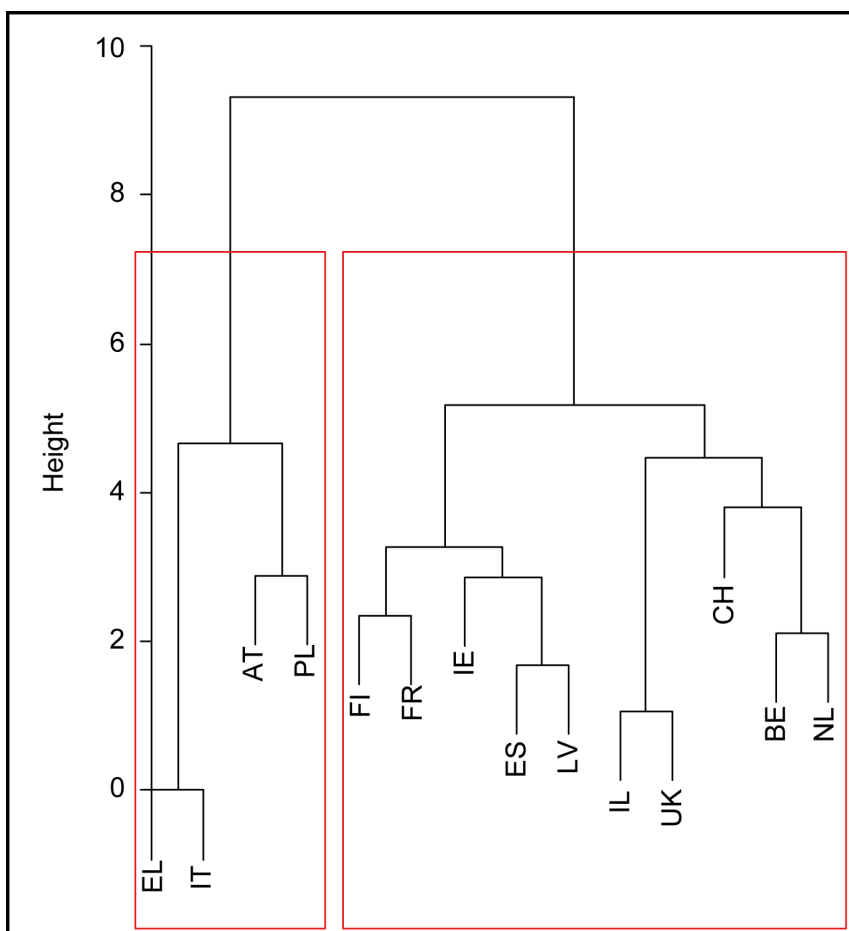

(a)

1 AT EL IT PL

2 BE CH ES FI FR IE IL LV NL UK

(b)
Silhouette plot of pam $(x=x d a t, k=i)$

$\mathrm{n}=14$

2 clusters $C_{j}$ $\mathrm{j}: \mathrm{n}_{\mathrm{j}}$ ave $_{\mathrm{i} \in \mathrm{C}} \mathrm{S}_{\mathrm{i}}$

1: $4 \mid 0.34$

2: $10 \mid 0.21$

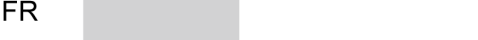

UK

$\mathrm{FI}$

$\mathrm{CH}$

$\mathrm{NL}$

PL

\begin{tabular}{cccccc}
\hline 0.0 & 0.2 & 0.4 & 0.6 & 0.8 & 1.0 \\
& & Silhouette width $\mathrm{s}_{\mathrm{i}}$ & &
\end{tabular}

(c)

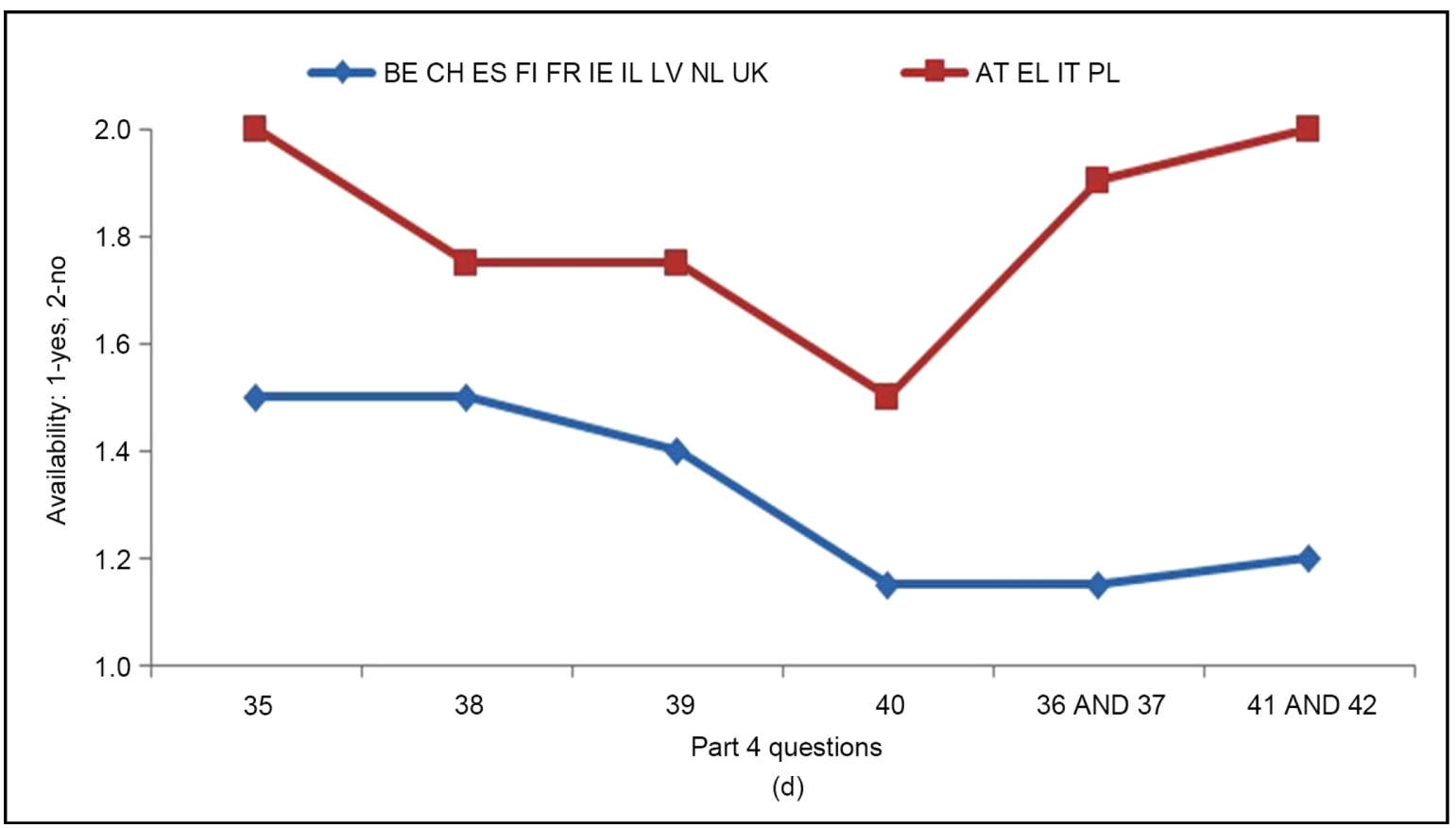

Figure 5. Result of countries' clustering using the "Monitoring and evaluation" characteristics: (a) a cluster dendrogram produced by the Ward method, (b) groups created by the k-means, (c) clusters' Silhouette produced by the PAM-method, (d) mean values of availability of the RSM components, by clusters of countries. 
the road safety situation; a sustainable system to collect and manage data on behavioural indicators is in place, where both some "process evaluation" of safety interventions during the implementation period of the program takes place and an evaluation of the effects on accidents and injuries of some policy components was planned. Other RSM components also tend to be available in these countries, where, however, in-depth accident investigations and a reporting procedure to monitor the road safety interventions carried out in the country are available in only half of the countries;

2 (AT, EL, IT, PL) - includes countries in which, in particular, in-depth accident investigations do not take place; the "process evaluation" of safety interventions during the implementation period of the program is not common; the evaluation of effects on accidents and injuries of policy components was not planned; and a sustainable system to collect and manage data on behavioural indicators is not in place (with the partial exception for AT). Also, other RSM components tend to be unavailable in these countries, except for the "benchmarking" relative to other (European) countries which is applied in some of them (PL, AT).

\subsubsection{Scientific Support, Information and Capacity Building}

Part 5 of the RSM questionnaire included 6 variables (having excluded two consensus variables, see Table A1). An initial analysis revealed certain correlations between the pairs of variables such as: 49 - 50, 44 and 46 (Pearson coefficients of 0.77 and 0.55, respectively, $\mathrm{p}<0.05)$. The values of these pairs were averaged and, hence, for the countries' clustering 4 variables remained.

The plots of criteria for selecting the number of clusters did not indicate a consistent choice. Accounting for the "bends" in the k-means plot, low points in the pseudo $\mathrm{T}$-squared plot and bigger between-group-distances on the Ward dendrogram we decided to examine 2 - 4 cluster solutions. A further analysis demonstrated higher consistency in the classification results received by different methods for the 3-clusters' solution, which, consequently, was selected for application. The classification results received by different methods are presented in Figure 6, as well as the mean values of cluster characteristics, based on Part 5 of the questionnaire.

It can be seen in Figure 6 that the Ward and the k-means methods produced identical results as to the composition of the three country groups. The Silhouette method recognized three homogeneous groups of countries which are generally similar to the results of other methods, except for the position of two countries: NL, ES; these two countries actually differ from other countries in their group and thus, could belong to other groups.

The countries' groups recognized using the "Scientific support and information, capacity building" components of the RSM questionnaire were:

1 (BE, FR, IL, IT, LV, UK) - comprises countries in which there are articles or programs in the media on road accidents and/or on road safety activities which review, criticize or challenge current policies, and where there is at least one university providing a multi-disciplinary course on road traffic safety for students. Other components of the RSM are also present, to some extent; 

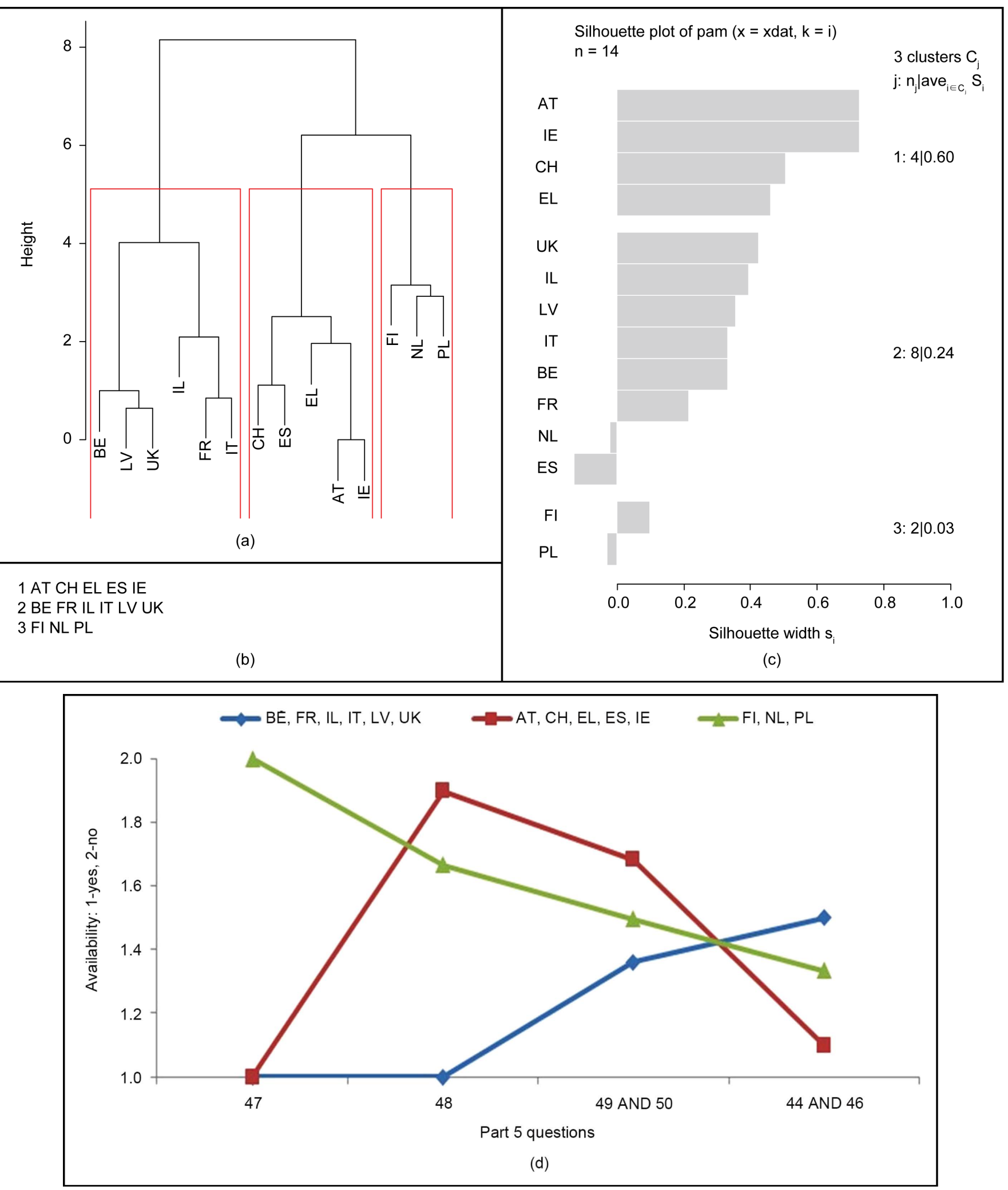

Figure 6. Result of countries' clustering using the "Scientific support, information and capacity building" characteristics: (a) a cluster dendrogram produced by the Ward method, (b) groups created by the k-means, (c) clusters' Silhouette produced by the PAM-method, (d) mean values of availability of the RSM components, by clusters of countries. 
2 (AT, CH, EL, ES, IE) - contains countries in which, similar to group 1, there are items in the media. In addition, in these countries stronger use of the road safety research results was reported, where the government or road safety institutions more systematically inform the citizens on the national road safety policy, interventions and their effects. On the other hand, in these countries no university provides a multi-disciplinary course on road safety;

3 (FI, NL, PL) -includes countries which, similar to group 2, tend to use the road safety research results, where the government or road safety institutions typically inform the citizens on the national road safety policy, interventions and their effects (especially true for NL). However, for these countries the experts reported on the absence of articles or programs in the media which would review, criticize or challenge current road safety policies.

One can notice that, in general, the level of availability of the Part 5 RSM components is close among the groups, where it is not obvious which group is clearly better in these terms.

\subsection{Comparison with Countries' Grouping Based on the Governmental Responses}

Similar data preparation and analyses were conducted using a set of 12 responses supplied by the governmental representatives of the countries (detailed results can be seen in [14]). Then, we examined differences in the countries' grouping based on the governmental versus expert responses. A first finding was that the list of "consensus variables" based on the governmental responses was longer, by four variables, relative to that based on the expert responses (which had seven consensus variables, see Table A1). This means that compared to the expert vision, the official one was more optimistic as it reported on the availability of more RSM components in the countries examined. The country officials in most countries believed that: a high level inter-sectoral decisionmaking institution was established in the country to prepare policy orientations for road safety; a national medium-term road safety program was elaborated in the country; a reporting procedure was set up to monitor the road safety interventions carried out in the country and "benchmarking" is commonly used to monitor progress in the road safety situation relative to other (European) countries, whereas experts doubted the availability of these components in the RSM systems of some countries. At the same time, both the experts and the officials agreed that, in most countries: there are some government agencies actively advocating the need for taking road safety action, and there are NGOs actively promoting road safety; national medium-term quantitative targets are set; a sustainable system to collect and manage data on road accidents, fatalities and injuries is in place; the government or road safety institutions provide factual information on road accidents, injuries and risk to the citizens, and there is at least one institute or university performing multi-disciplinary road safety research. In addition, both the experts and the officials reported that a budget needed to move towards longterm road safety vision is typically not estimated in the countries. The points of agree- 
ment between the two respondent groups strengthen the objectivity of findings and highlight the RSM components which are commonly available or unavailable, in the RSM systems of the developed countries.

Further clustering of the governmental responses with the purpose to recognize the countries' groupings according to various parts of the RSM questionnaire demonstrated that the results were usually different compared to those based on the expert responses. Only a few countries were classified similarly in both analyses whereas the majority of countries changed their positions on the cluster maps. Such a difference was (technically) expected given the various numbers of countries considered in both analyses, with sometimes different values assigned to the same characteristics. However, a more meaningful finding was that, in general, the governmental responses tend to state a higher availability of the RSM components in their countries compared to expert estimates.

A detailed comparison between the (50) answers provided by the experts versus the officials for each one of the countries demonstrated that in 8 cases (out 12) the values of experts were higher. Remarkably high differences in answers were found for such countries as IT, PL, UK, indicating that for these countries in many cases the official opinion was in favour of availability of certain RSM features, whereas experts doubted that. On the other hand, in two countries (IE, IL) the total score of differences was close to zero, pointing out a general similarity in the RSM system view, by both sides, where for one country (NL) the total score of differences was below zero, i.e. some expert estimates were more optimistic than that of the official.

Among the RSM components with stronger differences between both opinions, where the official responses systematically stated the availability of a feature and the expert not, were: a prominent role of Parliament in initiating decision-making on road safety orientations; carrying out consultations with local authorities (municipalities, counties) on the part they are called to play in national road safety policy; the application of a Safe-System approach while a national road safety strategy was produced; the availability of a national medium-term road safety program; the availability of formal resource allocation procedures to support RSM tasks and interventions; fund allocation to the evaluation of road safety interventions; setting up a reporting procedure to monitor the road safety interventions carried out in the country; setting up a procedure to evaluate safety performances of the global program; and regular informing the citizens, by the government or road safety institutions, on the national road safety policy and interventions and their effects.

In general, the data showed that governmental representatives tend to be more positive concerning the availability of the RSM components in their country, compared to the experts. Thus, in future analyses it might be better to use experts' opinions as the prime source.

\subsection{Final RSM Ranking of the Countries}

The next step was to summarize the results into a whole picture, in order to examine 
whether similarities can be seen among the countries, when the whole RSM structure is concerned. As expert responses covered a higher number of countries and, also, assuming that expert opinions reflect an independent and more objective view of the RSM system in the country, it was decided to identify the final country positions based on expert responses. Table 1 provides a summary of the groups each country belongs to, according to various aspects of the RSM. The group (cluster) numbering was given in such a way that the first cluster had the highest level of availability of the RSM components and the last-the lowest one. Based on the results of the five analyses, a final position (group) was estimated for each country, by means of rescaling and averaging the values. The countries were ranked in accordance with their final values, with possible alternatives of 3, 4 or 5 final country groups.

To recognize a high (or low) level of the RSM system as a whole, one could expect that countries will be systematically found in the first (or last) group of countries identified on the basis of each classification analysis. However, as Table 1 shows, the countries belong to various groups according to the results of analyses of various RSM parts. Indeed, a number of countries with a consistently higher level of the RSM component availability and with a consistently lower level of the same features can be recognized. Those are, for example, the groups of $\mathrm{CH}$, IL, FI and PL, EL, respectively. However,

Table 1. Country groups recognized in the analyses of five parts of the RSM questionnaire, final RSM country groups and country fatality rates.

\begin{tabular}{|c|c|c|c|c|c|c|c|c|c|}
\hline \multirow{2}{*}{ Country } & \multicolumn{5}{|c|}{$\begin{array}{l}\text { Country clusters identified following the RSM } \\
\text { questionnaire components' analysis of }\end{array}$} & \multicolumn{3}{|c|}{$\begin{array}{l}\text { A final RSM country group, where } \\
\text { the number of groups requested is }\end{array}$} & \multirow{2}{*}{$\begin{array}{l}\text { Fatality rate per } \\
\text { million inhabitants } \\
\text { of the country* }\end{array}$} \\
\hline & Part 1 & Part 2 & Part 3 & Part 4 & Part 5 & 3 & 4 & 5 & \\
\hline $\mathrm{AT}$ & 2 & 1 & 4 & 2 & 2 & 2 & 2 & 3 & 65.9 \\
\hline $\mathrm{BE}$ & 1 & 4 & 4 & 1 & 1 & 2 & 2 & 3 & 77.5 \\
\hline EL & 4 & 4 & 6 & 2 & 2 & 3 & 4 & 5 & 113.3 \\
\hline ES & 4 & 2 & 3 & 1 & 2 & 2 & 3 & 3 & 53.9 \\
\hline FI & 1 & 2 & 1 & 1 & 3 & 1 & 2 & 2 & 50.8 \\
\hline IT & 3 & 4 & 5 & 2 & 1 & 2 & 3 & 4 & 65.2 \\
\hline LV & 3 & 3 & 3 & 1 & 1 & 2 & 2 & 3 & 97.0 \\
\hline NL & 2 & 2 & 2 & 1 & 3 & 2 & 2 & 3 & 38.6 \\
\hline $\mathrm{PL}$ & 3 & 3 & 6 & 2 & 3 & 3 & 4 & 5 & 102.4 \\
\hline UK & 2 & 3 & 5 & 1 & 1 & 2 & 2 & 3 & 30.7 \\
\hline
\end{tabular}

${ }^{\star}$ Both RSM questionnaire responses and fatality rates referred to 2010 . 
even for the first countries' group with seemingly higher level of availability of the RSM components compared to others, it was not the best across all the examinations. As a result, where a final subdivision into 4 - 5 groups is considered (see Table 1), none of the countries belongs to the first group.

Another observation from Table 1 is that all the countries are different when the whole RSM systems are considered as no two countries belong to the same group according to the results of the five analyses. Our conclusion is that due to the diversity of existing forms of RSM in the countries examined, the task of identifying the typical RSM structures seems to be unrealizable when the RSM system is considered as a whole. However, it is possible to compare the countries when parts of the RSM system are considered separately as was demonstrated in this study. A diversity and complexity of the RSM schemes in European countries was also indicated by a recent study of Alfonsi et al. [21], who justified the need to focus on selected RSM tasks when comparing countries.

\subsection{Exploring a Statistical Link with Fatality Rates}

An assumption underlying the present research was that effective RSM organization is one of the conditions for obtaining good road safety results in a country [5] [14]. Thus, at the final study step, a statistical link was examined between the RSM clusters of countries and their safety performance, where the latter was characterized in terms of fatality numbers per million population of the country (see Table 1). Spearman's rank correlation coefficient was estimated, to explore the strength of association between two ranked variables, across various parts of the RSM questionnaire and final country groups-Table 2 .

The results showed that for most parts of the RSM questionnaire, except for part 5 "Scientific support and information, capacity building", a positive correlation was observed with the fatality rates of the countries. The strongest association with the fatality

Table 2. Spearman correlation coefficients for a relation between country fatality rate and the RSM clusters.

\begin{tabular}{cc}
\hline Issue of comparison: country clusters according to & Spearman correlation coefficient $(\mathrm{p}$-value $)$ \\
\hline Part 1 of the RSM questionnaire & $0.37(\mathrm{p}=0.19)$ \\
Part 2 of the RSM questionnaire & $0.48(\mathrm{p}=0.08)$ \\
Part 3 of the RSM questionnaire & $0.45(\mathrm{p}=0.11)$ \\
Part 4 of the RSM questionnaire & $0.63(\mathrm{p}=0.016)$ \\
Part 5 of the RSM questionnaire & $-0.028(\mathrm{p}=0.92)$ \\
Final RSM classification with three country groups & $0.63(\mathrm{p}=0.015)$ \\
Final RSM classification with four country groups & $0.48(\mathrm{p}=0.08)$ \\
Final RSM classification with five country groups & $0.57(\mathrm{p}=0.03)$ \\
\hline
\end{tabular}


rates was found for part 4 "Monitoring and evaluation": a correlation coefficient of 0.63 ( $\mathrm{p}<0.05$ ), while for part 2 "Policy formulation and adoption" and part 3 "Policy implementation and funding" the association was noticeable as well: correlation coefficients of $0.45-0.48$ (close to significant). In addition, a significant positive correlation was found between the fatality rates and final RSM country classifications: the correlation coefficients of $0.57-0.63(\mathrm{p}<0.05)$. Hence, our results indicate that a positive correlation does exist between the level of the RSM system in the country and its safety performance, where countries with higher availability of the RSM components were characterized by lower fatality rates.

However, admitting the small sample of countries examined in this study, we believe that a relationship between the RSM and country safety performance needs further investigation. Future research on the subject is particularly relevant accounting for insufficient findings of previous studies, e.g. [22] [23], where a direct relation between the RSM level and countries' safety outcomes (fatality rates) was not ascertained. Yet, Papadimitriou and Yannis [23] found a positive relationship between RSM and "intermediate outcomes" of the countries (indicators of driver behaviours, vehicle fleet, etc.).

\section{Conclusions}

Recognizing the importance of an effective RSM for promoting road safety at the country level [1] [5] [7], this study examined the presence of "good practice" components in the RSM systems of developed countries. Statistical classification techniques were applied to the responses provided to the RSM questionnaire [14], aiming to identify country groups with similar availability of certain RSM components and, if applicable, to recognize typical RSM structures in Europe and to show a relationship between RSM and countries' safety performance.

The analyses revealed that all the countries are different when the RSM systems are considered as a whole, making it impossible to identify typical RSM structures or a single best working model at a national level. However, it is possible to compare countries when the RSM areas are considered separately, where the clusters of countries recognized by the study actually present the patterns common for the European countries. Across the analyses, a number of countries with a consistently higher availability of some RSM components could be identified, and others with a consistently lower availability of the same features, providing a final countries' ranking into a number of groups. The latter actually reflects the level of RSM in the country, in terms of its correspondence to "good practice" criteria. A further indication of a correlation between a higher level of the RSM system and better safety performance of the country was also provided.

Among the countries, a higher availability level was observed for the presence of a strong lead agency, a national medium-term road safety program, quantitative targets, NGOs or government agencies actively advocating for taking road safety action, "benchmarking" progress related to other countries, systematic data collection, using research results and a media coverage of the road safety issues. Clearly, these "good prac- 
tice" features are common today for the RSM of the developed countries. On the other hand, low availability was found for most policy implementation and funding components, including a lack of dedicated budget, insufficiency of human resources, etc. The additional weak points of the RSM systems were: distribution and coordination of responsibilities between various management levels, and (un)availability of sustainable and results-focused structures which would enable effective implementation, funding, monitoring and evaluation of the road safety activities. The common patterns and particularities of the RSM systems revealed by the study pointed to the improvements needed for promoting better RSM in the European countries.

The RSM characteristics examined by this study referred to the year 2010, for which the questionnaire responses were collected [14]. Updated examinations of the RSM systems, carried out on a bigger country sample, would be useful in the future to provide a further exploration of the RSM structures and their over-time changes, and to verify a relationship between the RSM and countries' road safety progress.

\section{Acknowledgements}

This study was carried out within the framework of the DaCoTa research project of the $7^{\text {th }}$ Framework Programme of the European Commission/DG-Move. The authors are grateful to the DaCoTa WP1 group for providing valuable comments on this research.

\section{References}

[1] OECD (2008) Towards Zero: Ambitious Road Safety Targets and the Safe System Approach. Organization for Economic Co-Operation and Development, Paris.

[2] Elvik, R. (2008) Road Safety Management by Objectives: A Critical Analysis of the Norwegian Approach. Accident Analysis and Prevention, 40, 1115-1122. http://dx.doi.org/10.1016/j.aap.2007.12.002

[3] WHO (2013) Global Status Report on Road Safety 2013: Supporting a Decade of Action. World Health Organization, Geneva. http://www.who.int/violence_injury_prevention/road_safety_status/2013/en/index.html

[4] Bliss, T. and Breen, J. (2009) Implementing the Recommendations of the World Report on Road Traffic Injury Prevention. Country Guidelines for the Conduct of Road Safety Capacity Reviews and the Related Specification of Lead Agency Reforms, Investment Strategies and Safety Projects. World Bank Global Road Safety Facility, Washington DC.

[5] Muhlrad, N., Gitelman, V. and Buttler, I. (Ed.) (2011) Road Safety Management Investigation Model and Questionnaire. Deliverable 1.2 of the EC FP7 Project DaCoTA.

[6] OECD (2002) Road Safety: What's the Vision? Organisation for Economic Co-Operation and Development. OECD, Paris.

[7] ETSC (2006) A Methodological Approach to National Road Safety Policies. European Transport Safety Council, Brussels.

[8] Elvik, R. (1993) Quantified Road Safety Targets: A Useful Tool for Policy Making? Accident Analysis and Prevention, 25, 569-583. http://dx.doi.org/10.1016/0001-4575(93)90009-L

[9] Wong, S.C. and Sze, N.N. (2011) Is the Effect of Quantified Road Safety Targets Sustainable? Safety Science, 48, 1182-1188. http://dx.doi.org/10.1016/j.ssci.2009.12.020

[10] Muhlrad, N. (2005) Integrated Road Safety Management: Intersectoral Policies and Institu- 
tional Organisation. In: Tiwari, G., Mohan, D. and Muhlrad, N., Eds., The Way Forward: Transportation Planning and Road Safety, Macmillan India Ltd., Kolkata.

[11] Mulder, J. and Wegman, F. (1999) A Trail to a Safer Country: Conceptual Approach to Road Safety Policy. Proceedings of the 21 th World Road Congress, Kuala Lumpur, 3-9 October 1999.

[12] Jost, G., Allsop, R. and Steriu, M. (2012) A Challenging Start towards the EU 2020 Road Safety Target. 6th Road Safety PIN Report, ETSC, Brussels.

[13] Muhlrad, N., Vallet, G., Butler, I., Gitelman, V., Doveh, E., Dupont, E., Thomas, P., Talbot, R., Papadimitriou, E., Yannis, G., Giustiniani, G., Machata, K. and Bax, C. (2014) Analysis of Road Safety Management Systems in Europe. Transport Research Arena 2014, Paris.

[14] Papadimitriou, E., Yannis, G., Muhlrad, N., Gitelman, V., Butler, I. and Dupont, E. (Eds.) (2012) Analysis of Road Safety Management in the European Countries, Deliverable 1.5 Vol. 2 of the EC FP7 Project DaCoTA.

[15] Anderberg, M.R. (1973) Cluster Analysis for Applications. Academic Press, New York.

[16] Everitt, B., Landau, S., Leese, M. and Stahl, D. (2011) Cluster Analysis. 5th Edition, Wiley, Chichester. http://dx.doi.org/10.1002/9780470977811

[17] Xu, D. and Tian, Y.A. (2015) Comprehensive Survey of Clustering Algorithms. Annals of Data Science, 2, 165-193. http://dx.doi.org/10.1007/s40745-015-0040-1

[18] Everitt, B. and Hothorn, T. (2011) An Introduction to Applied Multivariate Analysis with R. Springer, New York. http://dx.doi.org/10.1007/978-1-4419-9650-3

[19] Murtagh, F. and Legendre, P. (2014) Ward's Hierarchical Agglomerative Clustering Method: Which Algorithms Implement Ward's Criterion? Journal of Classification, 31, 274295. http://dx.doi.org/10.1007/s00357-014-9161-z

[20] Rousseeuw, P.J. (1987) Silhouettes: A Graphical Aid to the Interpretation and Validation of Cluster Analysis. Journal of Computational and Applied Mathematics, 20, 53-65. http://dx.doi.org/10.1016/0377-0427(87)90125-7

[21] Alfonsi, R., Persia, L., Tripodi, A. and Usami, D.S. (2016) Advancements in Road Safety Management Analysis. Transportation Research Procedia, 14, 2064-2073.

[22] Elvik, R. (2012) Does the Use of Formal Tools for Road Safety Management Improve Safety Performance? Transportation Research Record: Journal of the Transportation Research Board, 2318, 1-6.

[23] Papadimitriou, E. and Yannis, G. (2013) Is Road Safety Management Linked to Road Safety Performance? Accident Analysis and Prevention, 59, 593-603.

http://dx.doi.org/10.1016/j.aap.2013.07.015 


\section{Appendix}

Table A1. Components of the RSM questionnaire and the final study dataset (based on expert responses).

\begin{tabular}{|c|c|c|c|c|c|c|c|c|c|c|c|c|c|c|c|}
\hline Part & Question & $\mathrm{AT}$ & $\mathrm{BE}$ & $\mathrm{CH}$ & EL & ES & FI & FR & IE & IL & IT & LV & NL & PL & UK \\
\hline 1 & $\begin{array}{l}\text { 2) Does Parliament have a prominent role in } \\
\text { initiating decision-making on RS orientations } \\
\text { or directions? }\end{array}$ & 2 & 1 & 1 & 2 & 1 & 1 & 1 & 2 & 2 & 1 & 1.5 & 2 & 2 & 1 \\
\hline 1 & $\begin{array}{l}\text { 3) Is Parliament involved in adopting RS } \\
\text { orientations or directions? }\end{array}$ & 2 & 1 & 1 & 2 & 2 & 1 & 2 & 1 & 2 & 1 & 1 & 1 & 1.5 & 1 \\
\hline 1 & $\begin{array}{l}\text { 4) Has a Lead Agency been formally appointed to } \\
\text { take responsibility for RS (direct the national RS } \\
\text { effort)? }\end{array}$ & 1 & 2 & 1 & 2 & 2 & 1 & 1 & 1 & 1 & 1 & 1 & 1 & 1 & 1 \\
\hline 1 & $6)^{\star}$ Coordination horizontally & 1.5 & 1 & 1.5 & 2 & 1.3 & 1.3 & 1.5 & 1.5 & 1.5 & 1 & 1 & 1.5 & 2 & 1.5 \\
\hline 1 & 7) Coordination vertically & 1.6 & 2 & 2 & 1.6 & 1.5 & 1 & 1.5 & 1.6 & 1 & 1.5 & 1.5 & 1 & 2 & 1 \\
\hline 1 & $\begin{array}{l}\text { 8) Has an institutional structure for the consultation } \\
\text { of stakeholders been formally established (by law or } \\
\text { decree)? }\end{array}$ & 1 & 1 & 1 & 1 & 2 & 1 & 1 & 2 & 2 & 2 & 2 & 1 & 2 & 1 \\
\hline 1 & $\begin{array}{l}9)^{\star} \text { Are the legislative instruments defining } \\
\text { inter-sectoral RS management functions } \\
\text { periodically reviewed and reformed? }\end{array}$ & 2 & 2 & 2 & 2 & 1.5 & 1 & 1.3 & 2 & 1.3 & 2 & 1.5 & 1.7 & 2 & 1 \\
\hline 2 & $\begin{array}{l}\text { 13) Are regional RS programs or policy components } \\
\text { integrated into the national RS policy? }\end{array}$ & 1 & 1 & 1 & 2 & 1.5 & 2 & 2 & 1.6 & 2 & 1 & 2 & 1 & 2 & 1.5 \\
\hline 2 & $\begin{array}{l}14)^{\star} \text { Are local authorities (municipalities, counties) } \\
\text { consulted as to the part they are called to play in } \\
\text { national RS policy? }\end{array}$ & 1 & 2 & 1 & 2 & 1.3 & 1 & 2 & 1.7 & 1.3 & 2 & 1.5 & 1 & 2 & 2 \\
\hline 2 & $\begin{array}{l}\text { 15) Are local RS programs or policy components } \\
\text { integrated into the national RS policy? }\end{array}$ & 1 & 2 & 1 & 2 & 2 & 1.5 & 2 & 2 & 2 & 1 & 1 & 2 & 2 & 2 \\
\hline 2 & $\begin{array}{l}\text { 16) Has a national "vision" for improved RS } \\
\text { performance in the long-term officially been set? }\end{array}$ & 1 & 1 & 2 & 1 & 1 & 1 & 1 & 2 & 1 & 1 & 2 & 1 & 2 & 2 \\
\hline 2 & $\begin{array}{l}\text { 17) }{ }^{\&} \text { Have national medium-term (four to ten years) } \\
\text { quantitative targets been set for improved } \\
\text { RS performance? }\end{array}$ & 1 & 1 & 2 & 1 & 1 & 1 & 1 & 1 & 1 & 1 & 1 & 1 & 1 & 2 \\
\hline
\end{tabular}




\section{Continued}

2 18) Has a national RS strategy been produced based on a Safe-System approach?

19) Has a national medium-term RS program been elaborated?

20) Has a national medium-term RS program been adopted at high level?

$21)^{*}$ Have partnerships or agreements been

3 established at the national level with the private sector?

22) If a national RS program has been elaborated

3 and adopted, has the budget needed for program implementation been estimated?

$23)^{\&}$ If a long-term vision has been adopted, has a budget been estimated to move towards this vision?

$34)^{*}$ Has a high-level engagement been taken to ensure availability of a budget for RS?

25) Does the government allocate the product of

3 fines (or any funds collected from RS measures) to RS interventions or related activities?

26) Is there a budget specifically allocated to RS

3 activities, interventions and capacity building from the national budget (Treasury)?

27) Is there a sustainable funding structure for RS, independent from the Treasury?

3 28) Are there formal resource allocation procedures to support RS management tasks and interventions?

3 29) Is funding allocated to evaluation?

$30)^{*}$ Are the funds allocated sufficient to implement

3 the program or policy components adopted in each area?

$31)^{*}$ Are the human resources needed to implement

3 the program or policy components adopted sufficient in each area? $32)^{\star}$ Are the legislative instruments and procedures regularly reviewed and improved?

33) Have training plans been designed to support

3 implementation of the national RS program or policy components?

$34)^{\&}$ Are sustainable systems in place to collect

4 and manage data on road accidents, fatalities and injuries? 


\section{Continued}

4 35) Are sustainable in-depth accident investigations

for RS purpose in place?

4 36) ${ }^{*}$ Are sustainable systems in place to collect and manage data on behavioural indicators?

4 37) Is there a national Observatory centralizing the data systems for RS?

38) Has a reporting procedure been set up to

4 monitor the RS interventions carried out in the country?

43) Has a procedure been set up to evaluate RS performances of the global program or policy?

40) Is "benchmarking" used to monitor progress

4 in the RS situation relatively to other (European) countries?

41) Does some "process evaluation" of safety

4 interventions take place during the implementation period of the program?

42) Has an evaluation process been planned to

4 assess the effects on accidents and injuries of some policy components ("product" evaluation)?

$543)^{\&}$ Is there at least one institute or university performing multi-disciplinary RS research?

$544)^{\star}$ Using research results for formulating RS policy

$45)^{\&}$ Are there government or RS institutions

5 providing factual and valid information on road accidents, injuries and risks to the citizens?

46) Are there government or RS institutions

5 systematically informing the citizens on the national RS policy, interventions and their effects?

47) Are there items in the media on road accidents

5 and/or on RS activities which review, criticize or challenge current policies?

5

48) Is there at least one university providing a multi-disciplinary course on RS for students?

$49)^{\star}$ Do universities offer specialized courses

5 addressing future professionals who may be involved in RS?

$50)^{\star}$ Do universities, research or other educational

5 institutions offer further-training sessions addressing key professionals currently involved in RS?

\begin{tabular}{|c|c|c|c|c|c|c|c|c|c|c|c|c|}
\hline 2 & 1 & 1 & 2 & 2 & 1 & 2 & 1 & 2 & 2 & 2 & 1 & 2 \\
\hline 1.3 & 1.3 & 1 & 2 & 2 & 1.3 & 1.3 & 1 & 1 & 2 & 1 & 1.3 & 2 \\
\hline 2 & 1 & 1 & 2 & 1 & 2 & 1 & 1 & 1 & 2 & 1 & 1 & 2 \\
\hline 2 & 2 & 2 & 2 & 1 & 1 & 1 & 1 & 2 & 2 & 1 & 2 & 1 \\
\hline 2 & 2 & 1 & 2 & 1 & 2 & 2 & 1 & 1 & 2 & 1 & 2 & 1 \\
\hline 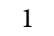 & 1 & 2 & 2 & 1 & 1 & 1 & 1 & 1 & 2 & 1.5 & 1 & 1 \\
\hline
\end{tabular}

2

2

$\begin{array}{rllllllllllll}2 & 1 & 2 & 1 & 1 & 1 & 1 & 1 & 2 & 1 & 1 & 2 & 1\end{array}$

$\begin{array}{lllllllllllllllll}1 & 1 & 1 & 1 & 1 & 1 & 1 & 2 & 1 & 1 & 1 & 1 & 1 & 1\end{array}$

$\begin{array}{llllllllllllll}1 & 1 & 1 & 1 & 1 & 1.5 & 2 & 1 & 1.5 & 1.5 & 1 & 1 & 1.5 & 1.5\end{array}$

$\begin{array}{llllllllllllll}1 & 1 & 1 & 2 & 1 & 1 & 1 & 1 & 1 & 1 & 1 & 1 & 1 & 1\end{array}$

$\begin{array}{lllllllllllllll}1 & 1 & 1 & 2 & 1 & 1 & 2 & 1 & 2 & 2 & 1.5 & 1 & 2 & 1\end{array}$

$\begin{array}{lllllllllllllllll}1 & 1 & 1 & 1 & 1 & 2 & 1 & 1 & 1 & 1 & 1 & 2 & 2 & 1\end{array}$

$\begin{array}{lllllllllllllll}2 & 1 & 2 & 2 & 1.5 & 2 & 1 & 2 & 1 & 1 & 1 & 1 & 2 & 1\end{array}$

$\begin{array}{llllllllllllll}1.7 & 1.4 & 1.3 & 2 & 1.4 & 1 & 1.5 & 1.7 & 1.1 & 1.6 & 1.4 & 1.7 & 1.6 & 1.3\end{array}$

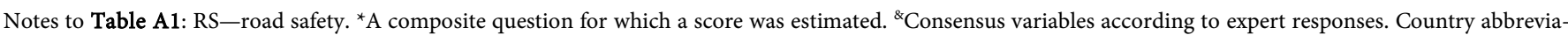

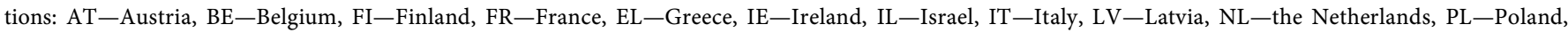
ES-Spain, $\mathrm{CH}$-Switzerland, UK-the United Kingdom. 
Submit or recommend next manuscript to SCIRP and we will provide best service for you:

Accepting pre-submission inquiries through Email, Facebook, LinkedIn, Twitter, etc. A wide selection of journals (inclusive of 9 subjects, more than 200 journals)

Providing 24-hour high-quality service

User-friendly online submission system

Fair and swift peer-review system

Efficient typesetting and proofreading procedure

Display of the result of downloads and visits, as well as the number of cited articles

Maximum dissemination of your research work

Submit your manuscript at: http://papersubmission.scirp.org/

Or contact jtts@scirp.org 